\title{
Hecke-Langlands Duality and Witten's Gravitational Moonshine
}

\author{
Igor Yu. Potemine \\ Institut de Mathématiques, Université Paul Sabatier, Toulouse, France \\ Email: igor.potemine@math.univ-toulouse.fr
}

How to cite this paper: Potemine, I.Yu. (2021) Hecke-Langlands Duality and Witten's Gravitational Moonshine. Journal of High Energy Physics, Gravitation and Cosmology, 7, 391-402. https://doi.org/10.4236/jhepgc.2021.72022

Received: December 31, 2020

Accepted: March 28, 2021

Published: March 31, 2021

Copyright (C) 2021 by author(s) and Scientific Research Publishing Inc. This work is licensed under the Creative Commons Attribution International License (CC BY 4.0).

http://creativecommons.org/licenses/by/4.0/

(c) (i) Open Access

\begin{abstract}
The purpose of this research is to give a dual description of conformal blocks of $d=2$ rational CFT (conformal field theory) in terms of Hecke eigenforms and eigensheaves. In particular, partition functions, conformal characters and lattice theta functions may be reconstructed from the action of Hecke operators. This method can be applied to: 1) rings of integers of Galois number fields equipped with the trace (or anti-trace) form; 2) root lattices of affine Kac-Moody algebras and WZW-models; 3) minimal models of Belavin-Polyakov-Zamolodchikov and related $d=2$ spin-chain/lattice models; 4) vertex algebras of Leech and Niemeier lattices and others. We also use the original Witten's idea to construct the 3-dimensional quantum gravity as the AdS/CFT-dual of $c=24$ Monster vertex algebra of Frenkel-LepowskyMeurman. Concerning the geometric Langlands duality, we use results of Beilinson-Drinfeld, Frenkel-Ben-Zvi, Gukov-Kapustin-Witten and many others ( $c f$. references). The main new result in this paper is the construction of number-theoretical lattice vertex superalgebras in Section 5 and applications to conformal field theories and quantum gravity.
\end{abstract}

\section{Keywords}

Conformal Field Theory, Modular Invariance, Quantum Gravity, $S$-Duality, Vertex Algebra

\section{Introduction: Number-Theoretical Dualities, $S$-Dualities and Gravitational Cosmology}

In this paper, we discuss an amazing interplay between number-theoretical dualites and black holes in Anti-de Sitter spaces. It passes through modular invariance of conformal field theories and AdS/CFT correspondences.

Recall that electric $E$ and magnetic field $B$ in the vacuum (in regions without charges and currents) are related by Maxwell equations, invariant by transfor- 
mations:

$$
E \mapsto B, B \mapsto\left(-1 / c^{2}\right) E
$$

where $c$ is the speed of light. This is the basic form of the electromagnetic duality. We can already interpret it as the modular $S$-invariance corresponding to the matrix $S=\left(\begin{array}{cc}0 & -1 \\ 1 / c^{2} & 0\end{array}\right)$, exchanging fields and reversing coupling constants. It extends to $S$-dualities in CFT and string theories.

In recent years [1] [2], it was realized that the Langlands correspondence is the number-theoretical counterpart of the electromagnetic duality in gauge field theories. Representations of the absolute Galois group correspond to modular objects on the moduli space of curves. Moreover, these modular objects are eigensheaves of Hecke operators with meaningful eigenvalues. In particular, the coefficients of the $L$-function

$$
L(E, s)=\sum_{n=1}^{\infty} \frac{a_{n}}{n^{s}}
$$

of an elliptic curve $E / \mathbb{Q}$ are Hecke eigenvalues, generating a cusp modular form

$$
f(E, q)=\sum_{n=1}^{\infty} a_{n} q^{n}
$$

of weight 2. This is a consequence of the famous modularity theorem related to the Fermat's Last Theorem.

In this paper, we define vertex operator algebras for all Galois number fields $K / \mathbb{Q}$, equipped with integral trace forms on their rings of integers $\mathcal{O}_{K}$ (cf. sect. 5). Thus, the modular $S$-invariance of lattice CFTs is closely related to the arithmetic Langlands program.

In addition, Witten [3] has associated, via AdS/CFT correspondence, the pure $d=3$ gravity to the Monster Moonshine Module $V^{*}$. It transports the Hecke-Langlands duality for the extremal $c=24$ CFT to modular dualities for BTZ black holes on $\mathrm{AdS}_{3}$ (cf. sect. 11).

The present paper is just a starting point of our research program. We describe the current state of art and define vertex superalgebras for number fields (cf. sect. 5) giving remarkable applications to CFT and quantum gravity.

\section{Virasoro Algebra and Rational CFT}

For general mathematical definitions, related to vertex algebras and conformal field theories (CFT), see [4] [5].

Recall that a vertex algebra $V=\oplus V_{n}$ is conformal of central charge $c \in \mathbb{C}$ if it contains a conformal vector $\omega \in V_{2}$ such that Fourier coefficients $L_{n}=L_{n}^{V}$ of the corresponding vertex operator

$$
Y(\omega, z)=\sum L_{n}^{V} z^{-n-2}
$$

satisfy the defining relations of the Virasoro algebra: 


$$
\left[L_{n}, L_{m}\right]=(n-m) L_{n+m}+c \frac{n^{3}-n}{12} \delta_{n,-m},\left[L_{n}, c\right]=0 .
$$

In addition, $L_{-1}^{V}=T: V \rightarrow V$ should be a translation operator of degree 1 , $\left.L_{0}^{V}\right|_{V_{n}}=n \cdot I d$ the grading operator and $L_{2} \omega=\frac{C}{2}|0\rangle$.

The Virasoro algebra is the central extension of $\mathbb{C}(t) \partial_{t}$ with topological basis given by $L_{n}=-t^{n+1} \partial_{t}, n \in \mathbb{Z}$, and $c \in \mathbb{C}$.

A conformal vertex algebra $\mathrm{V}$ is called rational if it is completely reducible with finitely many inequivalent simple modules having finite-dimensional graded components.

The basic example is given by the Virasoro vertex algebra $\operatorname{Vir}_{c}$ with central charge $c$ and conformal vector $\omega=L_{-2}|0\rangle$ ([4], sect. 2.5).

\section{Lattice Vertex Superalgebras}

Let $\Lambda$ be a lattice in $\mathbb{R}^{n}$ equipped with a non-degenerate $\mathbb{Z}$-valued symmetric bilinear form $\langle\cdot, \cdot\rangle$. There is a well-known quantization of the space of maps from the circle $S^{1}$ to the torus $\mathbb{R}^{n} / \Lambda$ called lattice vertex superalgebra.

Denote by $\mathfrak{h}=\mathbb{C} \otimes_{\mathbb{Z}} \Lambda$ the complexification of $\Lambda$ and $\mathfrak{h}^{<0}=\sum_{j<0} h \otimes t^{j}$. The space of state is

$$
V_{\Lambda}=\operatorname{Sym}\left(\mathfrak{h}^{<0}\right) \otimes C[\Lambda]
$$

where $C[\Lambda]$ is the group algebra of $\Lambda$, generated by $e^{\alpha} \quad(\alpha \in \Lambda)$. It is a superspace with parity $p\left(e^{\alpha}\right)=p(\alpha)=\langle\alpha, \alpha\rangle(\bmod 2)$.

For any $\alpha \in L$, mutually local fields $\Gamma_{\alpha}(z)=Y\left(1 \otimes e^{\alpha}\right)$ are of the form

$$
\Gamma_{\alpha}(z)=e^{\alpha} \exp \left(-\sum_{j<0} \frac{z^{-j}}{j} \alpha_{j}\right) \exp \left(-\sum_{j>0} \frac{z^{-j}}{j} \alpha_{j}\right) c_{\alpha} z^{\alpha_{0}}
$$

(cf. ([5], sect. 5.4)) with operators $c_{\alpha}$ on $V_{L}$ satisfying

$$
c_{0}=1, c_{\alpha}|0\rangle=|0\rangle,\left[h_{n}, c_{\alpha}\right]=0(h \in \mathfrak{h}, n \in \mathbb{Z})
$$

([5], (5.4.10)) and the locality condition

$$
e^{\alpha} c_{\alpha} e^{\beta} c_{\beta}=(-1)^{p(\alpha) p(\beta)+\langle\alpha, \beta\rangle} e^{\beta} c_{\beta} e^{\alpha} c_{\alpha}
$$

Together with free bosonic fields (for all $h \in \mathfrak{h}$ )

$$
Y\left(\left(h \otimes t^{-1}\right) \otimes 1, z\right)=h(z)=\sum_{n \in Z} h_{n} z^{-n-1}
$$

they generate a vertex operator algebra with the space of state $V_{L}$ and the vacuum vector $|0\rangle=1 \otimes 1$ ([5], prop. 5.4).

Putting $c_{\alpha}\left(s \otimes e^{\beta}\right)=c_{\alpha, \beta} s \otimes e^{\beta}$ for $s \in \operatorname{Sym}\left(\mathfrak{h}^{<0}\right)$ and $\beta \in \Lambda$, we get the following equations for numbers $c_{\alpha, \beta} \in \mathbb{C}$ :

$$
\begin{gathered}
c_{\alpha, 0}=c_{0, \beta}=1 \\
c_{\alpha, \beta}=(-1)^{p(\alpha) p(\beta)+\langle\alpha, \beta\rangle} c_{\beta, \alpha} \\
c_{\beta, \gamma} c_{\beta+\gamma, \alpha}=c_{\gamma, \alpha+\beta} c_{\beta, \alpha}
\end{gathered}
$$


It defines a cohomology class in $H^{2}\left(\Lambda, \mathbb{C}^{\times}\right)$, asserting the existence of a unique (up to isomorphism) vertex superalgebra structure on $V_{\Lambda}$ ([4], sect. 4.4.3).

Moreover, it is a rational conformal superalgebra with central charge $c=\operatorname{rank}(\Lambda)$.

\section{Lattice Theta Functions}

It can be shown (cf. ([4], sect. 4.5.5), ([6], p. 300)) that characters of lattice vertex algebras $V_{\Lambda}$ of even lattices $\Lambda$ are lattice theta functions (divided by $\eta(\tau)^{\text {rank } \Lambda}$ where $\eta(\tau)$ is the Dedekind $\eta$-function).

Let $\Lambda$ be an integral lattice of rank $n$. We define the theta series of $\Lambda$ as

$$
\Theta_{\Lambda}(q)=\sum_{v \in \Lambda} q^{\langle v, v\rangle / 2}=1+\sum_{k>0} N_{2 k}(\Lambda) q^{k}
$$

where $N_{k}(\Lambda)=\#\{v \in \Lambda \mid\langle v, v\rangle=k\}$. If $k>0$ is the minimal integer such that $N_{k}(\Lambda)>0$ then $N_{k}(\Lambda)$ is called the kissing number of $\Lambda$.

The lattice theta-series satisfies the functional equation

$$
\Theta_{\Lambda^{*}}\left(e^{-2 \pi t}\right)=\sqrt{\operatorname{disc}(\Lambda)} t^{-n / 2} \Theta_{\Lambda}\left(e^{-2 \pi / t}\right)
$$

where $\Lambda^{*}$ is the dual lattice and $\operatorname{disc}(\Lambda)=\#\left(\Lambda^{*} / \Lambda\right)$ is the discriminant of $\Lambda$.

Consider $\theta_{\Lambda}(\tau)=\Theta_{\Lambda}\left(e^{2 \pi i \tau}\right)$ as a function on the upper half-plane $\mathbb{H}_{+}$. If $\Lambda=\Lambda^{*}$ is self-dual (integral and unimodular) then we get functional equations:

$$
\theta_{\Lambda}(\tau+2)=\theta_{\Lambda}(\tau), \theta_{\Lambda}(-1 / \tau)=(\tau / i)^{n / 2} \theta_{\Lambda}(\tau) .
$$

Recall that the full modular group $\mathrm{PSL}_{2}(\mathbb{Z})$ is generated by matrices

$$
\begin{gathered}
S=\left(\begin{array}{cc}
0 & -1 \\
1 & 0
\end{array}\right) \text { and } T=\left(\begin{array}{ll}
1 & 1 \\
0 & 1
\end{array}\right) . \text { Then, for any } \gamma \in \Gamma_{+}=\left\langle S, T^{2}\right\rangle, \text { we have } \\
\theta_{\Lambda}(\gamma(\tau))=\varepsilon_{c, d}^{n}(c \tau+d)^{n / 2} \theta_{\Lambda}(\tau)
\end{gathered}
$$

So, $\left.\theta_{\Lambda}(\tau)\right)$ becomes a modular form of weight $n / 2$ for $\Gamma_{+}$with multiplier system $\varepsilon_{c, d} \in \mathbb{C}^{*}$ satisfying $\varepsilon_{c, d}^{8}=1$ (cf. ([7], sect. 2)).

Notice that the Dedekind $\eta$-function

$$
\eta(\tau)=q^{\frac{1}{24}} \prod_{n=1}^{\infty}\left(1-q^{n}\right)
$$

satisfies functional equations

$$
\eta(\tau+1)=\mathrm{e}^{\pi i / 12} \eta(\tau), \eta(-1 / \tau)=(\tau / i)^{1 / 2} \eta(\tau) .
$$

Thus, $\eta(\tau)$ is a modular form of weight $1 / 2$ and level 1 with a multiplier system of order 24 .

The general case of lattices with arbitrary discriminants, related to modular forms for congruence subgroups, needs additional investigations. However, when $\Lambda$ is an even lattice, the modularity follows from Zhu's results on characters of rational vertex algebras [6] (see sect. 6).

\section{Vertex Algebras for Number Fields}

Let $K=\mathbb{Q}[\xi]$ be a Galois number field over $\mathbb{Q}$ of degree $n=r_{1}+2 r_{2}$ where 
$r_{1}$ is the number of real embeddings and $2 r_{2}$ the number of complex embeddings of $K$ in $\mathbb{C}$. In this case of Galois extension over $\mathbb{Q}, K$ is either totally real $(\xi \in \mathbb{R})$ with $r_{2}=0$ or totally imaginary $(\xi \in \mathbb{C} \backslash \mathbb{R})$ with $r_{1}=0$.

The important thing here is to use the nondegenerate symmetric bilinear form

$$
\operatorname{tr}: K \times K \rightarrow \mathbb{Q},\langle x, y\rangle \mapsto \operatorname{tr}_{K / \mathbb{Q}}(x y),
$$

called the trace form. When $K$ is totally real, it is positive-definite. In the totally imaginary case, it is negative-definite and we should take rather the anti-trace form $\langle x, y\rangle \mapsto-t r_{K / \mathbb{Q}}(x y)$.

Let $\mathcal{O}_{K}$ be the ring of integers in $K / \mathbb{Q}$ corresponding to a lattice $\Lambda$, constructed from real and complex embeddings of $K$ (see ([8], ch. 1, section 1)). Recall that $\mathcal{O}_{K}$ is the unique maximal order in $K$. In our number-theoretical setting, there is a finite number of orders preserving $\Lambda$, equal to the class number $h(K)$ of $K$. The class number measures the deviation of $\mathcal{O}_{K}$ from being a principal ideal domain.

Our bilinear form $\langle\cdot, \cdot\rangle$ restricts to an integral form on $\mathcal{O}_{K}$. Now, choosing a basis $\left\{\omega_{1}, \cdots, \omega_{n}\right\}$ of $\mathcal{O}_{K}$, we can extend $\langle\cdot, \cdot\rangle$ to a positive-definite integral form on $\Lambda$.

So, we can construct a vertex superalgebra $\mathcal{V}_{K}$, associated to any Galois number field $K / \mathbb{Q}$. It opens the whole area of investigations in order to express the class field theory, aritmetical reciprocity laws etc. in terms of modularity theorems for lattice vertex superalgebras.

Consider a simple example of a real quadratic field $K=\mathbb{Q}(\sqrt{d})$ where $d$ is a square-free positive integer. Let

$$
\omega= \begin{cases}(1+\sqrt{d}) / 2 & d \equiv 1(\bmod 4) \\ \sqrt{d} & d \equiv 2,3(\bmod 4)\end{cases}
$$

Then $\{1, \omega\}$ is a basis of $\mathcal{O}_{K}$ ([8], ch. 1, ex. 2). An arbitrary order in $K$ is of the form $\mathcal{O}_{f}=\mathbb{Z}[f \omega]$ with a positive integer $f$ called the conductor of $\mathcal{O}_{f}$. If $\alpha=a+b \sqrt{d}$ then the trace $\operatorname{tr}_{K / \mathbb{Q}}(\alpha)=2 a$.

As we have seen in Section 3, the vertex superalgebra structure on $V_{\Lambda}$ is determined by a cocycle $c_{\alpha, \beta} \in H^{2}\left(\Lambda, \mathbb{C}^{\times}\right)$. Kac indicates an explicit form of this cocycle with values in $\mathbb{Z} / 2 \mathbb{Z}$ ([5], sect 5.5).

In order to be fully explicit, consider, for instance, $K=\mathbb{Q}[\sqrt{2}], \mathcal{O}_{K}=\mathbb{Z}[\sqrt{2}]$ and the corresponding even integral lattice $\Lambda=\mathbb{Z} \oplus \mathbb{Z} \sqrt{2} \in \mathbb{R}^{2}$ with basis $\{\alpha=(1,0), \beta=(0, \sqrt{2})\}$. Our bilinear form, associated to the trace form, gives

$$
\begin{gathered}
\langle\alpha, \alpha\rangle=\operatorname{tr}_{K / \mathbb{Q}}(1)=2 \\
\langle\alpha, \beta\rangle=\langle\beta, \alpha\rangle=\operatorname{tr}_{K / \mathbb{Q}}(\sqrt{2})=0 \\
\langle\beta, \beta\rangle=\operatorname{tr}_{K / \mathbb{Q}}(2)=4
\end{gathered}
$$

So, the matrix of the trace form is diagonal and discriminant $d(K)=2 \times 4=8$ as expected for this quadratic field. It is also well-known that $h(K)=1$.

Thus, the 2-cocycle of Frenkel-Kac, defining the unique (up to isomorphism) 
lattice vertex algebra $\mathcal{V}_{\mathbb{Z}[\sqrt{2}]}$ (with integral trace form), is given by the following formulas:

$$
\begin{gathered}
c_{\alpha, \alpha}=(-1)^{\left((\alpha, \alpha)+(\alpha, \alpha)^{2}\right) / 2}=(-1)^{\left(2+2^{2}\right) / 2}=-1 \\
c_{\alpha, \beta}=(-1)^{(\alpha, \beta)+(\alpha, \alpha)(\beta, \beta)}=1=c_{\beta, \alpha} \\
c_{\beta, \beta}=(-1)^{\left((\beta, \beta)+(\beta, \beta)^{2}\right) / 2}=(-1)^{\left(4+4^{2}\right) / 2}=1 .
\end{gathered}
$$

This vertex algebra has $d(K)=8$ inequivalent simple modules. Indeed, in the case of even integral lattices, Dong [9] has shown that inequivalent irreducible modules of $\mathcal{V}_{\Lambda}$ are parameterized by $\Lambda^{*} / \Lambda$.

Finally, let us notice that that the Fischer-Griess Monster group $F_{1}$ can be realized as a Galois group over $\mathbb{Q}$ ([10], p. 443, Case 1(b)). This is related to the Witten's gravitational moonshine considered in Section 11.

\section{Zhu's Modularity Results for Rational CFT}

Let $\mathcal{V}_{c}$ be a rational conformal vertex algebra of central charge $c$. For any simple $V_{c}$-module $M$ one can define its character by the formula

$$
\operatorname{ch} M=\operatorname{Tr}_{M} q^{L_{0}^{M}-c / 24}=\sum_{\alpha} \operatorname{dim} M_{\alpha} q^{\alpha-c / 24},
$$

where $M_{\alpha}$ is the subspace of $\mathrm{M}$ on which the Virasoro operator $L_{0}^{M}$ acts by multiplication by $\alpha$.

Let $C_{2}\left(\mathcal{V}_{c}\right)$ be the subspace generated by elements of the form $A_{(-2)} \cdot B$ for all $A, B \in \mathcal{V}_{c}$. Then $\mathcal{V}_{c}$ is said to satisfy Zhu's finiteness condition if $\operatorname{dim} \mathcal{V}_{c} / C_{2}\left(\mathcal{V}_{c}\right)<\infty$ and any vector can be written as $L_{n_{1}} \cdots L_{n_{k}} A, n_{i}<0$, where $L_{n} A=0$ for all $n>0$.

Theorem 6.1. ([6], thm. 5.3.2) Let $\mathcal{V}_{c}$ be a rational conformal vertex algebra of central charge $\mathrm{c}$ satisfying Zhu's finiteness condition. In addition, let

$\left\{M_{1}, \cdots, M_{m}\right\}$ be the complete list of irreducible $\mathcal{V}_{c}$-modules. Consider $n$-point correlation functions

$$
S_{M_{i}}\left(\left(a_{1}, z_{1}\right), \cdots,\left(a_{n}, z_{n}\right), \tau\right)
$$

as meromorphic continuations $\left(q=e^{2 \pi i \tau}\right)$ of limits

$$
\operatorname{Tr}_{M_{i}} Y\left(e^{2 \pi z_{1} L_{0}} a_{1}, e^{2 \pi z_{1} L_{0}}\right) \cdots Y\left(e^{2 \pi z_{1} L_{0}} a_{n}, e^{2 \pi z_{n} L_{0}}\right) q^{L_{0}-c / 24}
$$

If $a_{1}, \cdots, a_{n}$ are highest weight vectors with weights $w_{1}, \cdots, w_{n}$ for the Virasoro algebra, then for every $\left(\begin{array}{ll}a & b \\ f & d\end{array}\right) \in \mathrm{SL}_{2}(\mathbb{Z})$, we have

$$
\begin{gathered}
S_{M_{i}}\left(\left(a_{1}, \frac{z_{1}}{f \tau+d}\right), \cdots,\left(a_{n}, \frac{z_{n}}{f \tau+d}\right), \tau\right) \\
=(f \tau+d)^{\sum w_{k}} \sum_{j=1}^{m} S(\alpha, i, j) S_{M_{j}}\left(\left(a_{1}, z_{1}\right), \cdots,\left(a_{n}, z_{n}\right), \tau\right)
\end{gathered}
$$

where $S_{\alpha}(i, j)$ are constants depending only on $\alpha, i, j$. In particular, if $\mathcal{V}_{c}$ 
has a unique simple module $M$, and $a$ is a highest weight vector of weight $w$ for the Virasoro algebra, then $S_{M}(a, \tau)$ is a modular form of weight $w$ with a certain multiplier system.

Here we see the appearance of the modular $S$-matrix $S(\alpha, i, j)$ for any $\alpha \in \mathrm{SL}_{2}(\mathbb{Z})$. The functional equation for correlation functions corresponds to $\alpha=\left(\begin{array}{cc}0 & -1 \\ 1 & 0\end{array}\right)$.

Theorem 6.2. ([6], thm. 5.3.3) In the conditions of the previous theorem, ch $M_{i}$ converges to holomorphic functions on $\mathcal{H}_{+}$and the space, spanned by ch $M_{i}, 1 \leq i \leq n$, is invariant under the action of $\mathrm{SL}_{2}(\mathbb{Z})$.

\section{Minimal Models and $S$-Matrix of the Critical Ising Model}

It is known that $\operatorname{Vir}_{c}$ is reducible as the module over the Virasoro algebra if and only if

$$
c=c(p, r)=1-6(p-r)^{2} / p r, p, r>1,(p, r)=1
$$

In this case, the irreducible quotient $L_{c(p, r)}$ of $\operatorname{Vir}_{c(p, r)}$ is a rational vertex algebra, called minimal model of Belavin-Polyakov-Zamolodchikov [11] [12] [13].

In order to be explicit, consider the case $(p, r)=(4,3)$ with $c=1 / 2$. It is known as $d=2$ critical Ising model. There are 3 simple modules $M_{0}, M_{1 / 16}$ and $M_{1 / 2}$ with conformal dimensions $0,1 / 16$ and $1 / 2$ correspondings to primary fields 1 (identity), $\sigma$ (spin) and $\varepsilon$ (energy).

The characters are given by the following formulas $\left(q=e^{2 \pi i \tau}\right)$ :

$$
\begin{aligned}
& \operatorname{ch} M_{0}=\frac{\sqrt{\theta_{3}(0 \mid q)}+\sqrt{\theta_{4}(0 \mid q)}}{2 \sqrt{\eta(\tau)}} \\
& \operatorname{ch} M_{\frac{1}{16}}=\frac{\sqrt{\theta_{3}(0 \mid q)}-\sqrt{\theta_{4}(0 \mid q)}}{2 \sqrt{\eta(\tau)}} \\
& \operatorname{ch} M_{\frac{1}{2}}=\frac{\sqrt{\theta_{2}(0 \mid q)}}{\sqrt{2 \eta(\tau)}} .
\end{aligned}
$$

Here we used 3 remarquable theta functions (where $q=e^{\pi i \tau}$ ):

$$
\theta_{2}(0 \mid q)=\frac{2 \eta^{2}(2 \tau)}{\eta(\tau)}, \theta_{3}(0 \mid q)=\frac{\eta^{2}((\tau+1) / 2)}{\eta(\tau+1)}, \theta_{4}(0 \mid q)=\frac{\eta^{2}(\tau / 2)}{\eta(\tau)} .
$$

The functional equation for characters:

$$
\operatorname{ch} M_{i}(-1 / \tau)=\sum_{j} S(i, j) \operatorname{ch} M_{j}(\tau)
$$

is expressed in terms of the modular $S$-matrix:

$$
S(i, j)=\frac{1}{2}\left(\begin{array}{ccc}
1 & 1 & \sqrt{2} \\
1 & 1 & -\sqrt{2} \\
\sqrt{2} & -\sqrt{2} & 0
\end{array}\right) .
$$


corresponding to $\alpha=\left(\begin{array}{cc}0 & -1 \\ 1 & 0\end{array}\right)$ in the Zhu's theorem.

Finally, notice that the partition function

$$
Z(q)=\sum_{i}\left|\operatorname{ch} M_{i}\right|^{2}
$$

is modular invariant.

\section{Hecke Eigenforms and Wiles Modularity Theorem}

The mth Hecke operator $T_{m}$ acts on lattice functions by taking sums over all sublattices of index $m$. If $f(z)=\sum a_{n} q^{n}$ a weakly holomorphic (with possible poles at cusps) modular form of weight $k$ then

$$
\begin{aligned}
T_{m} f(z) & =n^{k-1} \sum_{a, d>0, a d=n} \frac{1}{d^{k}} \sum_{b \bmod d} f\left(\frac{a z+b}{d}\right) \\
& =\sum_{a d=n, \mu>0} a^{k-1} c_{d \mu} q^{a \mu}
\end{aligned}
$$

Denote $M_{k}$ the space of such weakly holomorphic modular forms and $S_{k}$ the space of cusp forms with $a_{0}=0$. Hecke operators transform cusp forms into cusp forms and, for a prime $m=p$, we get $T_{p}(f)=\sum b_{n} q^{n}$ with

$$
b_{n}= \begin{cases}a_{p n} & \text { if } p \nmid n \\ a_{p n}+p^{k-1} a_{n / p} & \text { if } p \mid n\end{cases}
$$

We can present $S_{k}$ as the direct sum $S_{k}^{\text {old }} \oplus S_{k}^{\text {new }}$ of subspaces of old and new cusp forms, orthogonal to each other with respect to the Petersson inner product. Old forms arise from modular forms of lower levels and have $a_{1}=0$. New forms with the same Diriclet character $\chi(\bmod N)$ are proportional and could be normalized by putting $a_{1}=1$. This is known as multiplicity 1 theorem.

Denote $\Gamma_{0}(N)$ the subgroup of matrices $\left(\begin{array}{ll}a & b \\ c & d\end{array}\right) \in \mathrm{SL}_{2}(\mathbb{Z})$ with $c \equiv 0(\bmod N)$ and consider the modular curve $X_{0}(N)=\Gamma_{0}(N) \backslash \mathbb{Z}_{+}$.

Theorem 8.1. (Wiles et al.) Let $E / \mathbb{Q}$ be a semi-stable elliptic curve with conductor $N$ and $L$-function

$$
L(E, s)=\sum_{n=1}^{\infty} \frac{a_{n}}{n^{s}} .
$$

Then there exists a new cusp Hecke eigenform $f(\tau)=\sum b_{n} q^{n}$ of weight 2 and level $N$ with $b_{n}=a_{n}$ (for almost all $n$ ), defining a modular parameterization $X_{0}(N) \rightarrow E \quad$ (up to an isogeny).

\section{Hecke Eigenfields and Maeda's Conjecture}

Let $f$ be a new cusp Hecke eigenform of level $N$ then

$$
T_{m} f(\tau)=\lambda_{m} f(\tau), \lambda_{m}=a_{m} a_{1}
$$

when $f(\tau)$ is normalized, we have $\lambda_{m}=a_{m}$.

Consider the field $K_{f}=\mathbb{Q}\left[\lambda_{m}, m \geq 1\right]$, generated by Hecke eigenvalues, that 
will be called Hecke eigenfield, associated to $f$. It is an algebraic extension of $\mathbb{Q}$.

Proposition 9.1. Let $N=1$. The Hecke eigenfield $K_{f}$ is totally real or CM-field (when $a_{1} \in \mathbb{C} \backslash \mathbb{R}$ ).

Proof. It follows from the fact that Hecke operators are self-adjoint with respect to the Petersson inner product.

Shimura has attached to $f(\tau)$ of weight 2 an abelian variety $S h_{f}$ of dimension $\left[K_{f}: \mathbb{Q}\right]$. In the Wiles case of elliptic curve $E / Q, K_{f}=\mathbb{Q}$ and $S h_{f}=E$ (up to an isogeny). Hecke eigenvalues correspond to the monodromy of the Galois action on $f(\tau)$ (as a horizontal section of an appropriate line bundle with a flat connection) around cusps. This is one of basic keypoints of the Beilinson-Drinfeld geometric Langlands duality.

The following Maeda's conjecture ([14], sect. 3.6.1) is important in order to study the Langlands duality in terms of Hecke eigenfields.

Conjecture 9.1. There is only one $\operatorname{Gal}(\overline{\mathbb{Q}} / \mathbb{Q})$-orbit of normalized eigenforms of level 1.

It is numerically proven for all $k \leq 4096$ [14].

\section{Critical WZW and Beilinson-Drinfeld Geometric Correspondence}

There are several books written on this topic [15] [16], so we briefly underline main ideas. It may be considered as the geometric $S$-duality for WZW-models for affine Kac-Moody algebras $\hat{G}$ at critical level $c=h^{\vee}$ (=dual Coxeter number of $G$ ).

Let $G$ be a reductive Lie group and ${ }^{L} G$ its Langlands dual (with dual root system). Denote $\operatorname{Bun}_{G}(X)$ the moduli space of principal $G$-bundles on a curve $X / \mathbb{C}, g(X) \geq 2$.

According to Beilinson and Drinfeld, there is a one-to-one correspondence

$$
\text { Hecke eigensheaves on } \operatorname{Bun}_{G}(X) \leftrightarrow{ }^{L} G \text {-local systems on } X
$$

Moreover, these Hecke eigensheaves are $D$-modules and their "eigenvalues" are exactly the corresponding ${ }^{L} G$-local systems. Via the Riemann-Hilbert correspondence, these local systems are representations of the fundamental group $\pi_{1}(X)$ playing the role of Galois representations.

Notice that geometric versions of Hecke operators are crucial for the geometric Langlands theory. A conjectural relationship with mirror symmetry was discussed in [17].

\section{Witten's Gravitational Moonshine}

Let $\mathcal{A}$ be a 3d-spacetime asymptotic at infinity to $\mathrm{AdS}_{3}$. Witten has suggested that the pure quantum gravity on $\mathcal{A}$ with maximally negative cosmological constant corresponds to an extremal $c=24$ two-dimensional CFT. This remarquable CFT was constructed by Frenkel-Lepowsky-Meurman [18] as the so-called Moonshine Module $V^{*}$ of the Monster vertex algebra $\mathbb{M}$. $\mathbb{M}$ is a rational vertex algebra and its unique irreducible representation $V^{*}$ is just $\mathbb{M}$ as 
module over itself. In this case there are 196883 operators, associated to Virasoro primary fields of $V^{*}$, creating BTZ black holes on $\mathcal{A}$ [3] [19].

The partition function of $c=24$ pure quantum gravity on $\mathrm{AdS}_{3}$ is simply

$$
Z_{1}(q)=J(q)=j(q)-744
$$

where $j(q)$ is the absolute $j$-invariant. Partition functions $Z_{k}(q)$ of hypothetical $c=24 k$ quantum gravities can be obtained using Hecke operators $T_{m}^{\prime}=m T_{m} \quad$ (or Faber polynomials $\Phi_{m}(X)$ such that $\Phi_{m}(j)=j_{m}(q)=T_{m}^{\prime}(J)$ [20]). Witten shows ([3], (3.13)) that $Z_{k}(q)$ should be of the form

$$
Z_{k}(q)=\sum_{m=0}^{k} a_{-m} T_{m}^{\prime} J(q)=\sum_{m=0}^{k} a_{-m} j_{m}(q)
$$

where $a_{-m}, 0 \leq m \leq k$, come from the formula

$$
q^{-k} \prod_{n=2}^{\infty}\left(1-q^{n}\right)^{-1}=\sum_{m=-k}^{\infty} a_{m} q^{m} .
$$

In this way we obtain

$$
Z_{2}(q)=\left(T_{2}^{\prime}+T_{0}^{\prime}\right) J(q)
$$

and so on. This construction is consistent with the Bekenstein-Hawking entropy for black holes. However, it is not yet clear to what extremal CFT correspond partitions functions $Z_{k}(q)$ for $k>1$.

It looks relatively simple at the level of partition functions but this is misleading. Zhu [6] shows that $V^{*}$ can be decomposed as the direct sum of tensor products of the highest weight modules of 48 Virasoro algebras with central charge $c=1 / 2$. Actually, it also proves the rationality of $\mathbb{M}$.

\section{Conclusions and Perspectives}

We have indicated just the beginning of the story. First of all, number-theoretical questions can be treated as a particular case of the study of lattice vertex superalgebras for number fields. It demonstrates an amazing unification of the arithmetic with lattice CFT theories.

On the one hand, arithmetic generalizations would include Drinfeld associators, motivic Galois groups and Grothendieck-Teichmüller groupoids. In positive characteristic, an upcoming article [21] will treat vertex $t$-algebras, generalizing Drinfeld modules and Anderson's $t$-motives [22].

On the other hand, physical generalisations would include the $S$-duality between electric and magnetic branes of the unifying $M$-theory. As Witten has already noticed, Hecke operators are related to Hooft operators and Wilson loops.

The holographic preimage of the Monstrous Moonshine, giving a spacetime with BTZ black holes, is inspiring and requires additional investigations.

\section{Acknowledgements}

I would like to thank my colleagues Vadim Schechtman and Joseph Tapia as well as my old friend Pavel Guerzhoy for inspiring discussions. 


\section{Conflicts of Interest}

The author declares no conflicts of interest regarding the publication of this paper.

\section{References}

[1] Gukov, S. and Witten, E. (2007) Gauge Theory, Ramification and Geometric Langlands Program. Current Developments in Mathematics, 2006, 35-180. https://doi.org/10.4310/CDM.2006.v2006.n1.a2

[2] Kapustin, A. and Witten, E. (2001) Electric-Magnetic Duality and the Geometric Langlands Program. Communications in Number Theory and Physics, 1, 1-236. https://doi.org/10.4310/CNTP.2007.v1.n1.a1

[3] Witten, E. (2007) Three-Dimensional Gravity Reconsidered. 1-82.

[4] Frenkel, E. and Ben-Zvi, D. (2001) Vertex Algebras and Algebraic Curves. American Mathematical Society, Providence.

[5] Kac, V. (1998) Vertex Algebras for Beginners (2nd Edition). American Mathematical Society, Providence. https://doi.org/10.1090/ulect/010

[6] Zhu, Y. (1996) Modular Invariance of Characters of Vertex Operator Algebras. Journal of the American Mathematical Society, 9, 237-302.

https://doi.org/10.1090/S0894-0347-96-00182-8

[7] Elkies, N. (2009) Theta Functions and Weighted Theta Functions of Euclidean Lattices, with Some Applications.

[8] Koch, H. (1992) Encyclopaedia of Mathematical Sciences. In: Parshin, A.N. and Shafarevich, I.R., Eds., Number Theory II, Springer, Berlin, 1-269.

[9] Dong, C. (1993) Vertex Algebras Associated with Even Lattices. Journal of Algebra, 161, 245-265. https://doi.org/10.1006/jabr.1993.1217

[10] Thompson, J. (1984) Some Finite Groups Which Appear as Gal $L / K$, Where $K \subseteq \mathbb{Q}\left(\mu_{n}\right)$. Journal of Algebra, 89, 437-499.

https://doi.org/10.1016/0021-8693(84)90228-X

[11] Belavin, A., Polyakov, A. and Zamolodchikov, A. (1984) Infinite Conformal Symmetries in Two-Dimensional Quantum Field Theory. Nuclear Physics B, 241, 333-380. https://doi.org/10.1016/0550-3213(84)90052-X

[12] di Francesco, P., Mathieu, P. and Senechal, D. (1997) Conformal Field Theory. Springer, Berlin. https://doi.org/10.1007/978-1-4612-2256-9

[13] Wang, W. (1993) Rationality of Virasoro Vertex Operator Algebras. International Mathematics Research Notices, 1993, 197-211.

[14] Ribet, K. and Stein, W. (2017) Lectures on Modular Forms and Hecke Operators.

[15] Beilinson, A. and Drinfeld, V. (2004) Chiral Algebras. American Mathematical Society, Providence. https://doi.org/10.1090/coll/051

[16] Frenkel, E. (2007) Langlands Correspondence for Loop Groups. Cambridge University Press, Cambridge.

[17] Potemine, I. (2001) Quantum Langlands Duality and Mirror Symmetry. 1-16.

[18] Frenkel, I., Lepowsky, J. and Meurman, A. (1988) Vertex Operator Algebras and the Monster. Academic Press, Cambridge. https://doi.org/10.1142/9789812798411 0010

[19] Ashrafi, M. (2020) Three Dimensional Gravity and the Generalized Hecke Operators. 1-20. 
[20] Guerzhoy, P. (2008) On Irreducibility of Certain Faber Plynomials. The Ramanujan Journal, 1, 53-57.

[21] Potemine, I. (2021) Vertex $t$-Algebras in Positive Characteristic.

[22] Potemine, I. (1999) Drinfeld-Anderson Motives and Multicomponent KP-Hierarchy. Recent Progress in Algebra: An International Conference on Recent Progress in Algebra, Taejon, 11-15 August 1997, Contemporary Mathematics 224, 213-227. 